\title{
Estudo retrospectivo da taxa de sobrevivência de implantes instalados por profissionais com diferentes graus de experiência na implantodontia
}

\author{
Retrospective study on the survival rate of implants installed by \\ professionals with different degrees of experience in implantology
}

Benedito Celso Ribeiro da Silva* Paulo Sérgio Perri de Carvalho* Eduardo Vedovato*** Ana Paula Farnezi Bassi ${ }^{* * * *}$ Jadison Junio Conforte ${ }^{* * * *}$ Daniela Ponzoni

\section{Resumo}

Objetivo: este estudo retrospectivo tem como objetivo avaliar a taxa de sobrevivência de implantes instalados por profissionais com diferentes graus de experiência na implantodontia. Materiais e método: foram analisados os prontuários clínicos de 612 pacientes (329 de um profissional experiente e 283 de alunos de uma pós-graduação lato sensu). Todos os pacientes receberam implantes de titânio da Conexão Sistemas de Prótese Ltda. (São Paulo, Brasil), no período de 2005 a 2010, e tinham suas próteses provisórias ou definitivas instaladas há pelo menos um ano. A taxa de sobrevivência dos implantes foi analisada considerando a região em que foram instalados. Resultados: um total de 1.640 implantes foi instalado em 612 pacientes, sendo 221 do gênero masculino e 391 do feminino. Do total de implantes instalados, 764 (46,59\%) foram feitos pelo profissional experiente e $876(53,41 \%)$ por alunos da pós-graduação. As taxas de sobrevivência comparadas entre os implantes instalados pelo profissional experiente e pelos alunos de pós-graduação foram de 96,99\% e 97,15\% respectivamente. Conclusão: este estudo retrospectivo permitiu concluir que houve semelhança entre as taxas de sobrevivência dos implantes instalados por um profissional experiente em comparação com os implantes instalados pelos alunos de um curso de especialização em Implantodontia.

Palavras-chave: Implantes dentários. Osseointegração. Sobrevivência. Qualificação profissional.

\section{Introdução}

Segundo a Academia Europeia de Periodontia, um implante pode ser considerado como sucesso se não apresentar mobilidade, infecção persistente ou dor quando em função, submetido às forças mastigatórias. Para se obter sucesso com implan-

Mestre em Implantodontia, Centro de Pós-Graduação São Leopoldo Mandic, Campinas, SP, Brasil.

Professora assistente doutora, Departamento de Cirurgia e Clínica Integrada da Faculdade de Odontologia de Araçatuba - Unesp, São Paulo, SP, Brasil. Acadêmico de Odontologia, Faculdade de Odontologia de Araçatuba - Unesp, São Paulo, SP, Brasil.

Professora assistente doutora, Departamento de Cirurgia e Clínica Integrada da Faculdade de Odontologia de Araçatuba - Unesp, São Paulo, SP, Brasil. 
te é necessário realizar sua instalação com técnica cirúrgica adequada, aguardar um longo tempo de cicatrização e fazer uma correta distribuição do estresse, quando em função ${ }^{1,2}$. Quando um implante em função não cumprir algum dos quesitos de sucesso, ele não deve ser considerado sobrevivente ${ }^{2-4}$.

Geralmente as perdas dos implantes endósseos orais ocorrem logo após a instalação devido à falta de estabilidade primária, que pode ser causada pela falta de experiência do cirurgião operador com pouca qualificação para exercer a especialidade ${ }^{5,6}$. Essa falta de estabilidade do implante acaba possibilitando a formação de um tecido conjuntivo fibroso entre o tecido ósseo e o implante ou, posteriormente, após ocorrer a osseointegração, devido ao trauma da sobrecarga oclusal, à infecção, ou as duas situações associadas?

Algumas doenças sistêmicas (como diabete e paciente irradiados) e/ou medicações (como os bifosfonatos) utilizadas para o tratamento ou controle dessas doenças podem comprometer o contato íntimo entre o implante e o tecido ósseo, antes de sua exposição ao meio oral ${ }^{8-10}$.

A variável sexo do paciente parece não ter influência sobre o insucesso dos implantes, mas problemas de saúde associados ao aumento da idade podem exigir modificações no plano de tratamento ${ }^{11}$. Esses pacientes podem vir a ter doenças crônicas e passar a usar medicações por longos períodos de tempo, o que pode influenciar negativamente, visto que quanto maior o tempo de tratamento, menor é a taxa de sucesso da reabilitação protética ${ }^{8,10,12}$.

Assim, vários são os fatores locais e sistêmicos que podem causar a falha dos implantes, e após a avaliação de anamnese e física, o paciente deve ser informado dos riscos antes da cirurgia de instalação dos implantes ${ }^{10}$.

As falhas nos implantes também podem aparecer na fase de instalação da reabilitação protética sobre esses, que geralmente é baseada em conceitos de próteses convencionais feitas sobre dentes e que podem gerar dúvidas na sobrevida dos implantes, fato justificado pelo pequeno número de estudos longitudinais com resultados clínicos e radiográficos dos diferentes tipos de reabilitações protéticas sobre implantes orais ${ }^{13,14}$.

O objetivo deste estudo foi avaliar retrospectivamente a taxa de sobrevivência dos implantes instalados por profissionais com diferentes graus de experiência na implantodontia.

\section{Materiais e método}

Este estudo foi aprovado pelo Comitê de Ética em Pesquisa da Faculdade de Odontologia e do Centro de Pesquisas Odontológicas São Leopoldo Mandic de Campinas, SP, sob o Protocolo $n^{\circ}$ 2011/0222.

Os critérios de inclusão dos pacientes no estudo foram:

a) saúde classificada como ASA I ou ASA II;

b) fumantes; c) implantes instalados em áreas com osso primitivo, enxertadas e em alvéolos pós-extração;

d) planejamento cirúrgico e protético realizado pela mesma equipe de profissionais;

e) implantes instalados do Sistema Conexão de Prótese Ltda.;

f) próteses sobre os implantes em função há, pelo menos, um ano.

Foram analisados prontuários de pacientes atendidos no curso de Especialização em Implantodontia do Núcleo de Educação Continuada em Odontologia (NEC Odonto) de Araçatuba, SP, Brasil, e em um consultório odontológico especializado em implantodontia, cujo profissional conta com experiência de mais de vinte anos na área, no período de 2005 a 2010. Os pacientes receberam os implantes e aguardaram o período de osseointegração de quatro meses na mandíbula e seis meses na maxila quando foram expostos ao meio bucal. Em seguida, as próteses foram confeccionadas. Todos os implantes e componentes protéticos eram da mesma marca comercial (Sistema Conexão de Prótese Ltda. ${ }^{\circledR}$, São Paulo, Brasil).

Os dados dos prontuários foram coletados e agrupados em: profissional que realizou a instalação do implante (profissional experiente ou aluno do curso de especialização), idade, sexo, queixa principal, comprometimento sistêmico, uso de tabaco.

Quadro 1 - Ficha utilizada para coleta de dados dos prontuários dos pacientes

Instalado por:
( ) profissional experiente
( ) aluno de especialização
Data da instalação:
Diâmetro:
Comprimento:
Região implantada:
( ) maxila anterior
( ) maxila posterior
( ) mandíbula anterior
( ) mandíbula posterior
Qualidade óssea:
( ) I
( ) Il
( ) III
( ) IV
( ) área enxertada
( ) osso remanescente
( ) insucesso. Provável causa:

Fonte: elaboração dos autores com base nos dados da pesquisa.

Os implantes foram considerados sobreviventes quando não apresentaram mobilidade, dor e 
estavam sustentando e retendo as próteses em função. Implantes que foram removidos ou perdidos por falta de osseointegração, doença peri-implantar ou alguma outra causa foram considerados como insucessos ${ }^{7}$. Os implantes que não estavam suportando ou retendo prótese foram excluídos do estudo.

Com relação aos implantes, foram coletadas informações sobre a localização (maxila, mandíbula, anterior ou posterior), tipo de osso receptor (qualidade óssea, área enxertada ou osso remanescente). Nos casos de insucesso, foram considerados os possíveis fatores responsáveis pela perda.

\section{Resultados}

Durante o período avaliado, de 2005 a 2010, o profissional experiente e os alunos da especialização atenderam 612 pacientes, 221 homens e 391 mulheres, que receberam 1.640 implantes, dos quais 48 foram perdidos, resultando em um índice geral de sobrevivência de $97,07 \%$ (Tabela 1 ).
Tabela 1 - Levantamento total dos implantes instalados e a taxa de sobrevivência dos implantes

\begin{tabular}{c|c|c|c}
\hline $\begin{array}{c}\text { Total de } \\
\text { implantes } \\
\text { instalados }\end{array}$ & Sobreviventes & Insucessos & $\begin{array}{c}\text { Taxa de } \\
\text { sobrevivência }\end{array}$ \\
\hline 1.640 & 1.592 & 48 & $97,07 \%$ \\
\hline \multicolumn{2}{|c|}{ Fonte: todas as tabelas são de elaboração dos autores com base nos dados }
\end{tabular}
da pesquisa.

O profissional experiente fez cirurgias para instalação de implantes em 329 pacientes, sendo 125 homens e 204 mulheres, que receberam 764 implantes, dos quais 23 foram perdidos, resultando em uma taxa de sobrevivência de 96,99\%. No mesmo período, os alunos da especialização (considerados com pouca experiência) do NEC Odonto fizeram cirurgias para instalação de implantes em 283 pacientes, sendo 96 homens e 187 mulheres. Foram realizados 876 implantes, dos quais 25 foram perdidos, resultando em uma taxa de sobrevivência de $97,15 \%$ (Tabela 2).

Tabela 2 - Comparação das taxas de sobrevivência dos implantes instalados pelos dois grupos

\begin{tabular}{l|c|c|c|c}
\cline { 2 - 4 } & Total de implantes instalados & Sobreviventes & Insucessos & Taxa de sobrevivência \\
\hline Profissional experiente & 764 & 741 & 23 & $96,99 \%$ \\
Alunos de especialização & 876 & 851 & 25 & $97,15 \%$ \\
\hline
\end{tabular}

O profissional experiente instalou 407 implantes na maxila, sendo que dezoito foram perdidos, resultando em índice de sobrevivência de 95,58\%; já na mandíbula ele instalou 357 implantes, sendo que cinco foram perdidos, com índice de sobrevivência de $98,6 \%$. Os alunos instalaram na maxila 387 implantes e onze foram perdidos, resultando em um índice de sobrevivência de $97,16 \%$, e na mandíbula foram instalados 489 implantes, dos quais catorze foram perdidos, com uma taxa de sobrevivência de $97,14 \%$ (Tabela 3 ).

Tabela 3 - Comparação das taxas de sobrevivência dos implantes de acordo com a localização da instalação: maxila ou mandíbula

\begin{tabular}{|c|c|c|c|c|c|c|}
\hline & $\begin{array}{c}\text { Número de } \\
\text { implantes instalados }\end{array}$ & $\begin{array}{c}\text { Região } \\
\text { implantada }\end{array}$ & $\begin{array}{c}\text { Número de } \\
\text { implantes instalados }\end{array}$ & Sobreviventes & Insucessos & $\begin{array}{c}\text { Taxa de } \\
\text { sobrevivência }\end{array}$ \\
\hline \multirow{2}{*}{$\begin{array}{l}\text { Profissional } \\
\text { experiente }\end{array}$} & \multirow{2}{*}{764} & Maxila & 407 & 389 & 18 & $95,58 \%$ \\
\hline & & Mandíbula & 357 & 352 & 5 & $98,60 \%$ \\
\hline \multirow{2}{*}{$\begin{array}{l}\text { Alunos de } \\
\text { especialização }\end{array}$} & \multirow{2}{*}{876} & Maxila & 387 & 376 & 11 & $97,16 \%$ \\
\hline & & Mandíbula & 489 & 475 & 14 & $97,14 \%$ \\
\hline \multirow{2}{*}{ Total geral } & \multirow{2}{*}{1640} & Maxila & 794 & 765 & 29 & $96,35 \%$ \\
\hline & & Mandíbula & 846 & 827 & 19 & $97,75 \%$ \\
\hline
\end{tabular}

Dos 764 implantes instalados pelo profissional experiente, 178 foram na maxila anterior, sendo que oito foram perdidos, resultando em um índice de sobrevivência de $95,5 \%$; já na maxila posterior, ele instalou 229 implantes, desses dez foram perdidos, com índice de sobrevivência de $95,63 \%$. Na mandíbula anterior, ele instalou 99 implantes e teve $100 \%$ de sobrevivência, já na mandíbula posterior, o profissional instalou 258 implantes, dos quais perdeu cinco, obtendo índice de sobrevivência de 98,06\%.
Os alunos instalaram na maxila anterior 175 implantes, e seis foram perdidos, resultando em um índice de sobrevivência de $96,57 \%$, na maxila posterior, eles instalaram 212 implantes, dos quais perderam cinco, obtendo um índice de sobrevivência de $97,64 \%$. Na mandíbula anterior, eles instalaram 165 implantes, dos quais apenas um foi perdido, tendo uma taxa de sobrevivência de $99,4 \%$, e na mandíbula posterior instalaram 324 implantes, sendo que treze foram perdidos, um índice de sobrevivência de 95,99\% (Tabela 4). 
Tabela 4 - Comparação das taxas de sobrevivência dos implantes de acordo com a localização da instalação

\begin{tabular}{|c|c|c|c|c|c|}
\hline & Região implantada & $\begin{array}{c}\text { Número de } \\
\text { implantes instalados } \\
\end{array}$ & Sobreviventes & Insucessos & $\begin{array}{c}\text { Taxa de } \\
\text { sobrevivência }\end{array}$ \\
\hline \multirow{4}{*}{$\begin{array}{l}\text { Profissional experiente } \\
764 \text { implantes }\end{array}$} & Maxila anterior & 178 & 170 & 8 & $95,50 \%$ \\
\hline & Maxila posterior & 229 & 219 & 10 & $95,63 \%$ \\
\hline & Mandíbula anterior & 99 & 99 & 0 & $100,00 \%$ \\
\hline & Mandíbula posterior & 258 & 253 & 5 & $98,06 \%$ \\
\hline \multirow{4}{*}{$\begin{array}{l}\text { Alunos de especialização } \\
876 \text { implantes }\end{array}$} & Maxila anterior & 175 & 169 & 6 & $96,57 \%$ \\
\hline & Maxila posterior & 212 & 207 & 5 & $97,64 \%$ \\
\hline & Mandíbula anterior & 165 & 164 & 1 & $99,40 \%$ \\
\hline & Mandíbula posterior & 324 & 311 & 13 & $95,99 \%$ \\
\hline \multirow{4}{*}{$\begin{array}{l}\text { Total geral } \\
1.640 \text { implantes }\end{array}$} & Maxila anterior & 353 & 339 & 14 & $96,03 \%$ \\
\hline & Maxila posterior & 441 & 426 & 15 & $96,60 \%$ \\
\hline & Mandíbula anterior & 264 & 263 & 1 & $99,62 \%$ \\
\hline & Mandíbula posterior & 582 & 564 & 18 & $96,91 \%$ \\
\hline
\end{tabular}

O profissional experiente instalou 764 implantes e perdeu 23, resultando em um índice de insucesso de $3,01 \%$, perdas que podem estar associadas a causas variadas (Tabela 5 ).

Tabela 5 - Fatores locais e/ou sistêmicos que podem estar associados com a perda dos implantes instalados pelo profissional experiente

\begin{tabular}{l|c}
\multicolumn{1}{c|}{$\begin{array}{c}\text { Fatores locais e/ou sistêmicos } \\
\text { profissional experiente }\end{array}$} & $\begin{array}{c}\text { Número de } \\
\text { implantes perdidos }\end{array}$ \\
\hline Tecido ósseo de baixa qualidade & 2 \\
Áreas enxertadas & 5 \\
Pouca altura e/ou espessura & 5 \\
Trauma cirúrgico & 5 \\
Implante imediato & 2 \\
Instalado no canal incisivo & 1 \\
Parafunção e doença psiquiátrica & 2 \\
Paciente fumante e diabético & 1 \\
Total de implantes perdidos & 23 \\
\hline
\end{tabular}

Os alunos do curso de especialização instalaram 876 implantes e perderam 25 , obtendo uma taxa de insucesso de $2,85 \%$, perdas que também podem estar associadas a várias causas (Tabela 6).

Tabela 6 - Fatores locais e/ou sistêmicos que podem estar associados com a perda dos implantes instalados pelos alunos de especialização

\begin{tabular}{|c|c|}
\hline $\begin{array}{l}\text { Fatores locais e/ou sistêmicos } \\
\text { alunos de especialização }\end{array}$ & $\begin{array}{l}\text { Número de } \\
\text { implantes perdidos }\end{array}$ \\
\hline Implante com hexágono danificado & 1 \\
\hline Parestesia & 2 \\
\hline $\begin{array}{l}\text { Implante imediato com mobilidade e } \\
\text { secreção }\end{array}$ & 1 \\
\hline $\begin{array}{l}\text { Implante curto / 3,75 x 8,5 em região } \\
\text { posterior }\end{array}$ & 1 \\
\hline Dentro do seio maxilar & 2 \\
\hline Em contato com dente adjacente & 2 \\
\hline Mal posicionado & 1 \\
\hline Trauma da prótese total imediata & 1 \\
\hline Paciente diabético & 2 \\
\hline Mobilidade/paciente diabético & 1 \\
\hline Área enxertada/paciente diabético & 2 \\
\hline Paciente fumante & 1 \\
\hline $\begin{array}{l}\text { Levantamento de seio maxilar/ } \\
\text { paciente fumante }\end{array}$ & 1 \\
\hline $\begin{array}{l}\text { Implante imediato/paciente fumante } \\
\text { e diabético }\end{array}$ & 1 \\
\hline Paciente com anemia & 2 \\
\hline $\begin{array}{l}\text { Não associados a qualquer fator } \\
\text { específico }\end{array}$ & 4 \\
\hline Total de implantes perdidos & 25 \\
\hline
\end{tabular}




\section{Discussão}

Poucos estudos são relatados na literatura comparando o nível de experiência entre os profissionais que instalam implantes. Lambert et al. ${ }^{6}$ (1997) demonstraram que implantes instalados por cirurgiões inexperientes tinham duas vezes mais chance de falhar em relação aos instalados por um profissional experiente. Porém, Kohavi et al. ${ }^{15}$ (2004) relataram que a experiência clínica parece não ser um fator influente na sobrevivência dos implantes, desde que haja monitoramento dos níveis de formação profissional e também que os alunos, durante $o$ curso, tenham acompanhamento e recebam orientação constante de professores experientes. Esse fator parece ter contribuído para que os índices de sobrevivência dos implantes instalados, tanto pelo profissional experiente quanto pelos profissionais com pouca experiência no ramo, fossem próximos.

Alunos dos cursos de especialização em implantodontia no Rio Grande do Sul e em Santa Catarina instalaram 370 implantes do Sistema Conexão e, após o período de osseointegração, avaliaram a estabilidade secundária, obtendo um índice de sucesso de $97,57 \%$, que foi obtido por meio do contratorque de $25 \mathrm{Ncm}$ no momento da reabertura dos implantes para a instalação dos cicatrizadores ${ }^{16}$. Taxa de sucesso semelhante ao resultado dos alunos de especialização deste estudo $(97,15 \%)$.

Em um estudo recente de Zupnik et al. ${ }^{17}$ (2011), os autores acompanharam estudantes residentes em Periodontia da Harvard School of Dental Medicine, de Boston, por um período de quatro anos, quando foram instalados 341 implantes com um índice de sucesso de $96,48 \%$, valores que foram obtidos na avaliação da reabilitação protética. $\mathrm{O}$ índice de sucesso dos alunos residentes foi similar ao encontrado na literatura e não foi relevante o tempo de residência dos alunos, de um, dois ou três anos.

Apesar do pequeno número de trabalhos na literatura abordando a experiência profissional como possível fator de risco de perda de implantes, a maioria afirma que a curva de aprendizado desenvolvida nesses anos para a técnica de instalação de implantes parece ter sido fundamental para que os índices de sucesso fossem muito semelhantes entre os diversos níveis de experiência, quase sempre acima de $90 \%$ e muito próximos aos índices de sucesso obtidos no presente estudo, que foram de $96,99 \%$ para o profissional experiente e de $97,15 \%$ para os alunos de especialização.

Os índices obtidos neste trabalho são semelhantes aos de estudos pesquisados na literatura, como o de Adell et al. ${ }^{1}$ (1981), que acompanharam por quinze anos, de 1965 a 1980, 2.768 implantes do sistema Bränemark, que haviam sido instalados em 410 maxilares totalmente desdentados de 371 pacientes, obtendo uma taxa de sucesso de $89 \%$ na maxila e de $100 \%$ na mandíbula, em que o sucesso era considerado a partir da estabilidade protética.

Definir as causas das perdas dos implantes é muito difícil, pois na maioria das vezes elas podem ser multifatoriais. A maioria das perdas dos implantes instalados pelo profissional experiente, vinte em 23 , parece estar associada a cirurgias com maior grau de dificuldade, casos mais complexos, envolvendo regiões com tecido ósseo de baixa qualidade (perda de dois implantes), áreas enxertadas (perda de cinco implantes), perdas que são explicadas devido ao fato de o osso neoformado geralmente ser de baixa qualidade, tipo IV, o que dificulta a estabilidade inicial do implante ${ }^{4,18}$, osso com pouca altura e/ou pouca espessura (perda de cinco implantes), trauma cirúrgico (perda de cinco implantes), implante imediato após exodontia (perda de dois implantes) e instalação de implante no canal incisivo (perda de um implante). Em apenas dois casos a perda parece estar relacionada ao estado de saúde dos pacientes (ASA II), um apresentava parafunção e doença psiquiátrica (perda de dois implantes), condição que é contraindicada para terapia com implantes ${ }^{11}$, e a outra paciente (perda de um implante) era fumante e diabética (ASA II), condições em que o sucesso de implantes tem uma taxa mais baixa ${ }^{8,10,13,18}$.

Os 25 implantes perdidos nos pacientes dos alunos parecem estar associados a causas variadas, e alguns sem causa definida. As perdas ocorreram em algumas fases do tratamento, sendo:

a) um implante foi removido na cirurgia, pois o hexágono estava defeituoso e não foi possível instalar outro no local;

b) dez implantes foram perdidos no período de reparação óssea, dois implantes devido à parestesia e um implante imediato com mobilidade e secreção que podem ter sido perdidos devido à pouca experiência cirúrgica dos cirurgiões $^{5,6,15,17}$, um implante com mobilidade em paciente diabético (ASA II), que tem índice de sucesso menor que em paciente normal ${ }^{8,10,13}$, um implante curto de 3,75 x 8,5 em região posterior, que pode ter sido perdido devido à inexperiência do operador, visto que a literatura mostra índice de sucesso de 96,46\% em mandíbulas atróficas ${ }^{19}$, um implante em paciente com anemia (ASA II) e quatro implantes que não estavam associados a qualquer fator específico;

c) sete implantes foram perdidos na cirurgia de reabertura: um implante estava dentro do seio maxilar e um implante em contato com o dente adjacente, que podem ter sido perdidos devido à pouca experiência cirúrgica dos cirurgiões ${ }^{5,6,15,17}$, um implante instalado em região de levantamento de seio, cujo paciente era fumante ${ }^{18,20}$, dois implantes em paciente diabético e dois implantes em área enxertada com biomaterial em paciente diabético (ASA II). Pacientes nessas condições, fumante e dia- 
bético, apresentam menor índice de sucesso do que pacientes sem essas características ${ }^{8,10,13}$, embora em uma revisão de literatura ${ }^{9}$ concluiu-se que diabetes não deve ser considerada como uma contraindicação absoluta para a terapia com implantes, entretanto, deixar de fumar aumenta muito o índice de sobrevivência dos implantes ${ }^{5}$

d) sete implantes foram perdidos na fase de reabilitação protética: um implante estava dentro do seio maxilar, um implante estava em contato com o dente adjacente, um implante estava mal posicionado, um implante que parece ter sofrido trauma da prótese total imediata, perdas que devem ter ocorrido devido à pouca experiência dos cirurgiões, que podem ter errado na execução das cirurgias para a instalação de implantes e nos controles de pós-operatório ${ }^{5,6,21-23}$, um implante imediato em paciente fumante e diabético (ASA II), um implante em paciente fumante e um implante em paciente com anemia (ASA II). Algumas doenças sistêmicas e/ou as medicações utilizadas para o tratamento ou controle dessas doenças podem comprometer o contato íntimo entre o implante e o tecido ósseo, antes de sua exposição ao meio oral ${ }^{8-10}$.

Quanto à região da instalação dos implantes, os índices de sobrevivência obtidos pelo profissional experiente e pelos alunos foram respectivamente: na maxila anterior $95,5 \%$ e $96,57 \%$, na maxila posterior $95,63 \%$ e $97,64 \%$, na mandíbula anterior $100 \%$ e $99,4 \%$ e na mandíbula posterior $98,06 \%$ e $95,99 \%$. Esses índices são muito similares aos índices de sucesso obtidos por Souza $^{9}$ (2010), que avaliou 753 implantes do sistema Conexão em um estudo retrospectivo de um a oito anos. O autor observou taxas de sucesso de 95,8\% na maxila anterior, $93,8 \%$ na maxila posterior (menor índice), $100 \%$ na mandíbula anterior e 96,2\% na mandíbula posterior.

A semelhança nos índices de sobrevivência dos implantes entre os dois grupos avaliados neste estudo retrospectivo pode estar relacionada com a grande evolução, nos últimos anos, da técnica do protocolo cirúrgico para instalação de implantes dentais, o que permite que mesmo profissionais sem experiência, uma vez que respeitem os princípios cirúrgicos, obtenham índices de sucesso semelhantes aos de um profissional experiente ${ }^{6,17,21-23}$. A melhoria na macro e na micro estrutura dos implantes otimizou seus desempenhos, em especial na estabilidade primária e na aceleração do processo de osseointegração assim como a seleção mais criteriosa dos pacientes candidatos à reabilitação com implantes ${ }^{12,15}$.

A proximidade dos resultados obtidos nos grupos estudados realça que se o protocolo de planejamento cirúrgico-protético for criterioso e acompanhado por professores experientes, a experiência profissional torna-se um fator irrelevante na reabilitação oral com implantes.

\section{Conclusão}

Este estudo retrospectivo permitiu concluir que houve semelhança entre as taxas de sobrevivência dos implantes instalados por um profissional experiente, em comparação com os implantes instalados pelos alunos de um curso de especialização em Implantodontia.

\section{Abstract}

Objective: This retrospective study aimed to assess the survival rate of implants installed by professionals with different degrees of experience in Implantology. Materials and method: Clinical records of 612 patients were analyzed (329 of experienced professionals and 283 of post-graduate students). All patients had received titanium implants from Conexão Sistema de Prótese Ltda. (São Paulo, Brazil) from 2005 to 2010, and had their temporary or definitive prostheses installed for at least one year. Implants survival rate was analyzed considering the region where they were installed. Results: $A$ total of 1,640 implants were installed in 612 patients, wherein 221 were men and 391 were women. From the total of implants installed, 764 (46.59\%) were made by experienced professionals and $876(53.41 \%)$ by post-graduate students. The survival rates compared between implants installed by experienced professionals and by post-graduate students were $96.99 \%$ and $97.15 \%$, respectively. Conclusion: This retrospective study concluded there was similarity between the survival rates of implants installed by experienced professionals when compared to implants installed by post-graduate students in Implantology.

Keywords: Dental implants. Osseointegration. Survival rate. Professional qualification.

\section{Referências}

1. Adell R, Lenkholm U, Rockler B, Branemark PI. A 15-year study of osseointegrated implants in the treatment of the edentulous jaw. Int J Oral Surg 1981; 10(6):387-416.

2. Faverani LP, Ramalho-Ferreira G, Gaetti-Jardim EC, Okamoto R, Shinohara EH, Assunção WG et al. Implantes osseointegrados: evolução e sucesso. Salusvita 2011; 30(1):4758.

3. Galvão FFSA, Almeida-Júnior AA, Faria-Júnior NB, Caldas SGFR, Reis JMSN, Margonar R. Previsibilidade de implantes curtos: revisão de literatura. RSBO 2011; 8(1):81-8.

4. Luciano AA, Griza GL, Magro Filho O, Werlang JFG, Pavelski MD. Estudo clínico retrospectivo da taxa de sucesso precoce de implantes osseointegrados. Dent Press Implantol 2013; 7(3):76-83.

5. Preiskel HW, Tsolka P. Treatment outcomes in implant therapy: the influence of surgical and prosthodontic experience. Int J Prosthodont 1995; 8(3):273-9.

6. Lambert PM, Morris HF, Ochi S. Positive effect of surgical experience with implants on second-stage implant survival. J Oral Maxillofac Surg 1997; 55(12 Suppl 5):12-8. 
7. Albrektsson T, Isidor F. Consensus report of session IV. In: Lang NP, Karring T. Proceedings of the $1^{\text {st }}$ European Workshop on Periodontology. London: Quintessence 1994; 365-9.

8. Bornstein MM, Cionca N, Mombelli A. Systemic conditions and treatment as risks for implant therapy. Int J Oral Maxillofac Implants 2009; 24(Suppl):12-27.

9. Mombelli A, Cionca N. Systemic disease affecting Osseointegration therapy. Clin Oral Implant Res 2006; 17(Suppl 2):97-103.

10. Moy PK, Medina D, Shetty V, Aghaloo TL. Dental implant failure rates and associated risk factors. Int J Oral Maxillofac Implants 2005; 20(4):569-77.

11. Sugerman PB, Barber MT. Patient selection for endosseous dental implants: oral and systemic considerations. Int $\mathrm{J}$ Oral Maxillofac Implants 2002; 17(2):191-201

12. Canullo L, Cicchese P, Sisti A, Francischone Junior CE, Francischone CE, Vasconcelos LW et al. Análise clínica retrospectiva (4 - 6 anos) dos implantes P-I Branemark Philosophy. Implant News 2009; 6(5):517-24.

13. Wood MR, Vermilyea SG. Committee on Research in fixed prosthodontics of the academy of fixed prosthodontics. A review of selected dental literature on evidence-based treatment planning for dental implants: report of Committee on research in fixed prosthodontics of the Academy of Fixed Prosthodontics. J Prosthet Dent 2004; 92(5):447-62.

14. Blanes RJ, Bernard JP, Blanes ZM, Belser UC. A 10-year prospective study of ITI dental implants placed in the posterior region. II: Influence of the crown-to-implant ratio and different prosthetic treatment modalities on crestal bone loss. Clin Oral Implant Res 2007; 18(6):707-14.

15. Kohavi D, Azran G, Shapira L, Casap N. Retrospective clinical review of dental implants placed in a university training program. J Oral Implantol 2004; 30(1):23-9.

16. Silva LB, Poger W, Derech ED, Bortoluzzi MC, Manfro R. Avaliação da estabilidade secundária de 370 implantes consecutivos do Sistema Conexão. Implant News 2011; 8(3):377-80

17. Zupnik J, Kim SW, Ravens D, Karimbux N, Guze K. Factors associated with dental implant survival: a 4-year retrospective analysis. J Periodontol 2011; 82(10):1390-5.

18. Souza JGO. Influência dos fatores locais e sistêmicos na osseointegração e perda óssea ao redor de implantes: estudo retrospectivo de 1 a 8 anos [Dissertação de Mestrado]. Florianópolis: Universidade Federal de Santa Catarina; 2010.

19. Melhado RMD, Vasconcelos LW, Francischone CE, Petrilli CQG. Avaliação clínica de implantes curtos $(7 \mathrm{~mm}$ ) em mandíbulas. Acompanhamento de dois a 14 anos. Implant News 2007; 4(2):147-51.

20. Manfro R, Nascimento Junior WR, Loureiro JA, Bortoluzzi MC, Zago CRD. Avaliação do sucesso de 20 casos de levantamentos de seio maxilar utilizando osso autógeno particulado e Gen-Ox inorgânico associados em partes iguais (1:1) controle de dois anos. Implant News 2009; 6(2):161-6.

21. Ferreira LCP, Brito CR, Lehn CN, Siqueira JTT. Avaliação de implantes osseointegráveis submetidos à função imediata comparados à tardia. Implant News 2010; 7(1):13-20. Ilus, tab, graf.

22. Camacho FMT, Sakakura CE, Mera MFM, Esteves JC, Ribeiro FS, Pontes AEF. Avaliação clínica em curto prazo do sistema de Cone Morse e plataforma reduzida em prótese sobre implantes do tipo protocolo: estudo piloto randomizado controlado. Rev Odontol Unesp 2012; 41(4):247-53.

23. Nóia CF, Ortega-Lopes R, Moraes M, Albergária-Barbosa JR, Moreira RWF, Mazzonetto R. Complicações decorrentes do tratamento com implantes dentários: análise retrospectiva de sete anos. Rev Assoc Paul Cir Dent 2010; 64(1):55-8.
Endereço para correspondência:

Jadison Junio Conforte

Faculdade de Odontologia de Araçatuba - Unesp

Rua José Bonifácio, 1.193

16015-050 - Araçatuba, SP

Telefone: (18) 98200-5758

E-mail: junioconforte@yahoo.com.br

Recebido: 30/12/14. Aceito: 10/09/15. 